\title{
A comprehensive study on eye issues and modern developments in visual rehabilitation for people with impaired vision
}

\begin{abstract}
The eye is an important part of the human body and is a window that reveals the outside world to mankind. The eye is divided into anterior and posterior sections, each of which contains specific features and diseases. One of the most important problems with the human eye is blindness that can have hereditary or acquired causes. Many diseases, including fetal measles, retinopathy in premature infants, microphthalmosis, cataracts, etc. can cause blindness, and diabetes is one of the most important causes of blindness in the world. In this review study, we describe and review the features and components of the eye structure, familiarity with the concept of blindness, the diseases that cause blindness, and systems designed for blind use such as the white cane, the braille line, the finger reader technology, the guiding dogs, the money recognition device, the vibrotac technology, etc.
\end{abstract}

Volume 5 Issue 2 - 2019

\author{
Hamidreza Shirzadfar, Parisa Amirzadeh \\ Department of Biomedical Engineering, Sheikhbahaee University, \\ Isfahan, Iran
}

\begin{abstract}
Correspondence: Hamidreza Shirzadfar, Department of Biomedical Engineering, Sheikhbahaee University, Isfahan, Iran, Email h.shirzadfar@shbu.ac.ir,h.shirzadfar@gmail.com
\end{abstract}

Received: February 04, 2019 | Published: April 30, 2019

Keywords: cataracts, microphthalmosis, retinopathy, fetal measles, diabetes, hereditary causes, blindness

\section{Introduction}

The human eye forms the basis of the visual process, and the vision process involves a set of structures that lie alongside each other. The crystalline lens focuses the light beams through a process called controlled placement through the cellular muscle, which is a very necessary operation, also there is a neural network for processing and transmitting electrical signals received through the optic nerve and transferring them to the visual cortex of the brain in the mechanism of vision. As shown in Figure 1, the eye is composed of many components and is generally divided into anterior and posterior sections, the anterior part including the cornea, conjunctiva, iris, anterior chamber, eyelashes frame, eclipse, lens, etc. The back includes sclera, vitreous, choroid, optic nerve, and retina. ${ }^{1-3}$ When we remove the eye from the orbit, we can see that the eye is a slightly asymmetrical sphere with an approximate sagittal diameter or length of 24 to $25 \mathrm{~mm}$ and a transverse diameter of $24 \mathrm{~mm}$. It has a volume of about $6.5 \mathrm{cc}$ (Figure 1). 4,5 The external layer, formed by the sclera and cornea. The intermediate layer, divided into two parts: anterior (iris and ciliary body) and posterior (choroid). The internal layer or the sensory part of the eye is the retina. Three chambers of fluid: anterior chamber (between cornea and iris), posterior chamber (between iris, zonule fibers and lens), and the vitreous chamber (between the lens and the retina). The first two chambers are filled with aqueous humor, whereas the vitreous chamber is filled with a more viscous fluid, the vitreous humor. The sagittal section of the eye also reveals the lens, which is a transparent body located behind the iris. The lens is suspended by ligaments (called zonule fibers) attached to the anterior portion of the ciliary body. The contraction or relaxation of these ligaments, as a consequence of ciliary muscle actions, changes the shape of the lens, a process called accommodation that allows us to form a sharp image on the retina. ${ }^{5}$ In the following each section is generally explained:

I. Iris: This section includes two layers, first the pigmented fibrovascular layer and second layer is the pigmented epithelia cell. The iris makes the space between the cornea and the lens divide into 2 parts. The first is the anterior compartment and the second is the back compartment. The color we see in the iris depends on the individual's genetics, and in general, this color is created genetically. The muscles in the iris tissue react with the sympathetic and parasympathetic nerves and shrink and expand, causing the pupil to tighten or dilate.

II. Cornea: This part is located on the front of the eyeball and has no blood vessels to feed, but is fed by tears and the aqueous humor. The cornea has two important tasks, the first is to send the light into the eye, centralizing them on the retina, and the latter is to protect the eyes from the risks that may occur to them. The structure of the cornea is organized in five layers by cells and proteins.

III. Conjunctiva: It is a thin, transparent membrane that lies inside the eyelid and covers the front part of the sclera and protects the eyes against the micro-organisms by producing the mucosal matter. The conjunctiva consists of three parts: the first is the conjunctiva of the eyelid, which is a thin layer and covers the upper and lower eyelids. The latter is a bulbous conjunctiva that covers the sclera and the third is the split conjunctiva, a part that connects the conjunctiva of the eyelid and the bulbous conjunctiva.

IV. Anterior chamber: It is a part of the eye that contains aqueous humor $(\sim 0.25 \mathrm{ml})$ and is approximately $3 \mathrm{~mm}$ deep and is located on the front of the iris. There is a method for testing the anterior chamber, called a gonioscopy method that measures the automatic eye movements and the eye rotation.

V. Eyelashes frame: It is a thick tissue of the intravascular layer in the eye which centralizes the epithelium by lens cellular muscle to help the secretion of the aqueous humor.

VI. Aqueous humor: It is a transparent liquid that fills the anterior and posterior chambers and feeds the cornea. Aqueous humor acts like blood for these structures. Disposes the wastes from the metabolism, as well as the displacement of neurotransmitters, in addition, stabilizes the structure of the eye and helps to maintain 
the pressure of the eye. A large part of the aqueous humor is made up of water and there is a very small amount of protein in it.

VII. Lens: The part that lies behind the pupil and, in addition to being transparent, it becomes thin or thick with respect to the distance between objects that are close or far from the eye, and it is also flexible. The lens is responsible for focusing the light on the retina so that we can see the right and clear image.

VIII. Vitreous: It is a clear, jelly-like liquid that does not have any color and is located in the space between the lens and the retina and fills the space. Vitreous forms a huge amount of the eye and about $98 \%$ of the vitreous is water and the other $2 \%$ contains Hyaluronic acid and collagen fibers. There are no blood vessels in the vitreous.

IX. Chloride: It is a vascular layer that feeds the retina and supplies it with oxygen. It has two interior and exterior surfaces, the inner surface of which is connected to the retina pigment epithelium, and the outer surface is connected to the light nerves connected to the sclera and exits through the vortex vessels. A large portion of the chloride is the melanocyte cells; the blackness of this layer is due to the presence of these melanocytes.

X. Sclera: It is the part that forms the whiteness of the human eye and consists of collagen fibers. If the sclera is blurring, it's because collagen fibers are set irregularly. The task of the sclera is to protect the eyes, stabilizing the eye and to maintain the appearance of the eye. The sclera is divided into three parts: Epi-sclera, Lamina fusca and sclera stroma.

XI. Retina: It is the inner layer in the eye which is a circular disk with a diameter of approximately $30-40 \mathrm{~mm}$ (Kolb 1991) and a thickness of $0.5 \mathrm{~mm}$. These cells are divided into three main groups: photo receptors, neuronal cells and glial cells.

XII. Optic nerve: This nerve connects the eye to the brain and is called a cranial nerve. The nerve consists of ganglion cells with 1.2 million neurons and its task is to transmit visual waves from the retina and transmit them through the electrical waves to the center of the imaging in the brain. The mentioned contents are the only important parts of the eye structure because the eye structure has many complex components.

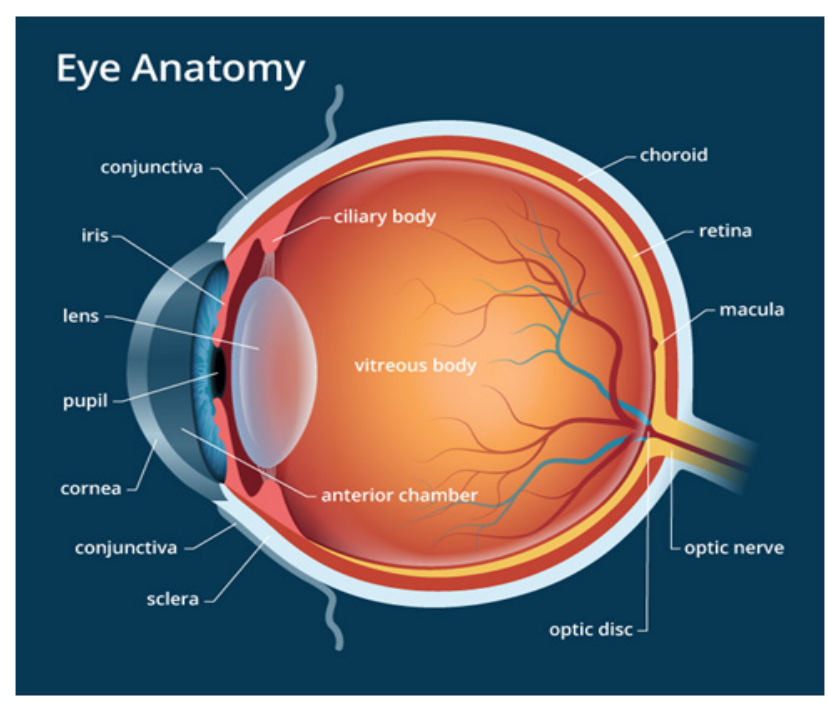

Figure I The human eye components. ${ }^{4}$

\section{Eye diseases and illnesses}

\section{Blindness}

Legally in the UK, someone who is unable to do things in which vision is needed is considered as a blind. If the patient's vision is below the mentioned level, then that person is considered blind. For people with visual impairments, social, financial and cultural services are considered that they can use these services if they so wish. Another definition of blindness is that a person with one of the following two characteristics is blind: $:^{6-9}$

A. The visibility of a person is less than 20degrees.

B. A person's field of view is less than 20degrees.

It should be noted that the blindness standards for different individuals can vary from year to year.

\section{Color blind}

These people are not blind because people who are color blind are unaware of this subject until they have tested their vision and surroundings. About $8 \%$ of men have congenital color blindness. ${ }^{10,11}$

\section{Causes of blindness}

In the Western world, the cause of blindness in children is central nervous system disorders, hereditary retinal dystrophies and congenital anomalies, and in adults aged between 20 and 60years, the main cause of retinal disease is diabetic retinopathy and optic atrophy. The causes of blindness are different in different parts of the world, including Africa and Asia. Many children suffer from blindness due to measles at the fetal period. The most common cause of blindness is cataract in adults. In parts of the world that are economically high, the problems of the blind are different from those that are poor and economically at a low level. There are many causes of blindness that are generally divided into two categories: the inherited causes, and the acquired causes. A series of illnesses that cause blindness is illustrated below:

Infection of the measles during fetal development: If the mother has measles infection during the first 12 weeks of pregnancy, her baby is likely to develop congenital measles, which may cause many problems for the child, one of which is eye defects, which can lead to blindness. ${ }^{12-19}$

Retinopathy in preterm infants: The disease is caused by the retinal development disorder that, if the baby achieves excessive oxygen, due to the advanced development of new vessels in the retina, retinal detachment ultimately leads to blindness. As shown in Figure 2, retinopathy in preterm infants. ${ }^{20}$

a) Stage 1: This is where the junction of the central part of the retina that already has blood vessels and the peripheral part of the retina where blood vessels have not yet grown is marked by a demarcation line or a flat white line. Babies with stage 1ROP usually recover without needing any treatment.

b) Stage 2: In stage 2, the demarcation line is raised and is more like a ridge. Babies with stage 2 ROP may recover without needing any treatment as well. These babies may require more regular check-ups to monitor progress.

c) Stage 3: Stage 3 is where new blood vessels start to grow out of the ridge. These blood vessels are very weak and they will cause vision problems if they start to scar. If there is also enlargement and tortuosity of the main normal blood vessels of the retina, 
this is known as "plus disease". Stage 3 ROP with plus disease usually requires treatment.

d) Stage 4: In stage 4, the fragile new blood vessels that grow in stage 3 can contract (shrink) causing scarring which can pull the retina away from the back of the eye (partial retinal detachment).

e) Stage 5: Stage 5 is where the retina can become totally detached from the back of the eye (total retinal detachment). This would lead to permanent loss of vision. ${ }^{21}$

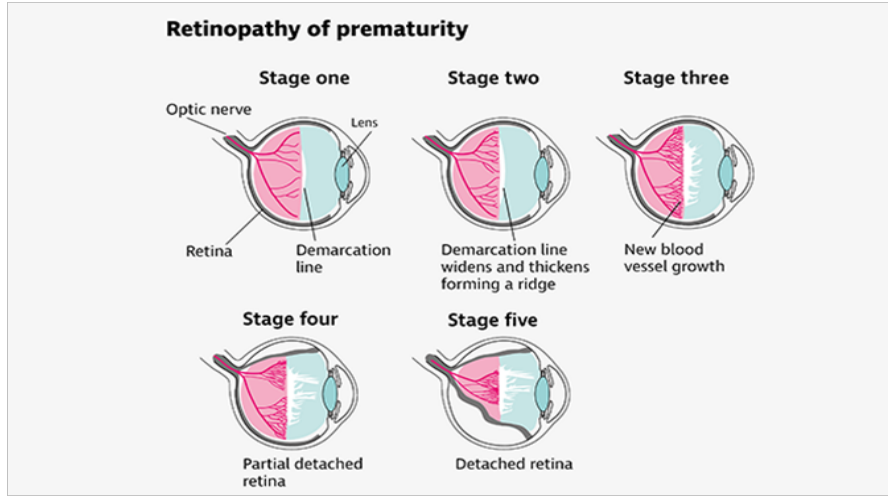

Figure 2 Stages of the Abnormal growth of the blood vessels causing the detachment of the retina from the back of the eye. ${ }^{21}$

Retinopathy of prematurity (ROP) is a disorder of the developing retina of low birth weight preterm infants that potentially leads to blindness in a small but significant percentage of those infants. ${ }^{22}$ The proportion of blindness as a result of ROP varies greatly among countries, being influenced both by levels of neonatal care (in terms of availability, access, and neonatal outcomes) and by the availability of effective screening and treatment programs. This raises important questions concerning strategies to reduce the incidence of blindness as a result of ROP, which should include ensuring that all infants who are at risk are examined in screening programs. ${ }^{23}$

\section{Microphthalmos}

The disease occurs due to maternal infections during pregnancy, cytomegalovirus infection, hepatitis, infections such as trans-plasmosis or hereditary and genetic transmission, and alcohol consumption during pregnancy and abnormal chromosomes. As shown in Figure 3, the eyeball of the fetus and the born child has structural problems and defects that cause the child to have limited vision. ${ }^{24-27}$

Glaucoma or the black water: If for any reason the balance between the production and excretion of the aqueous humor collapses, the amount of the aqueous humor in the eye increases and the intraocular pressure increases more than the normal. An increase in the pressure of the eye damages the retina and the nerve of the eyes and causes the black water. ${ }^{28-30}$

Cataracts: One of the main causes of blindness in the world that can occur in all ages, but with age increment, in addition to diminishing luminosity, lens opacity also diminishes and it also is a common symptom of intraocular inflammation. Sometimes the intensity of the lens blurring increases to a degree that looks like a curtain in front of the person's eyes. The created opacity may occur due to its position, for example, in the axis of the vision or at the anterior lens..$^{31,32}$

According to the Figure 4 these clumps gradually get larger and interfere with vision. ${ }^{33}$ They distort or block the passage of light through the lens. "Cataract" means "huge waterfall" or "enormous downpour," which is how some people describe their clouded sight, like trying to look through a waterfall. ${ }^{31}$
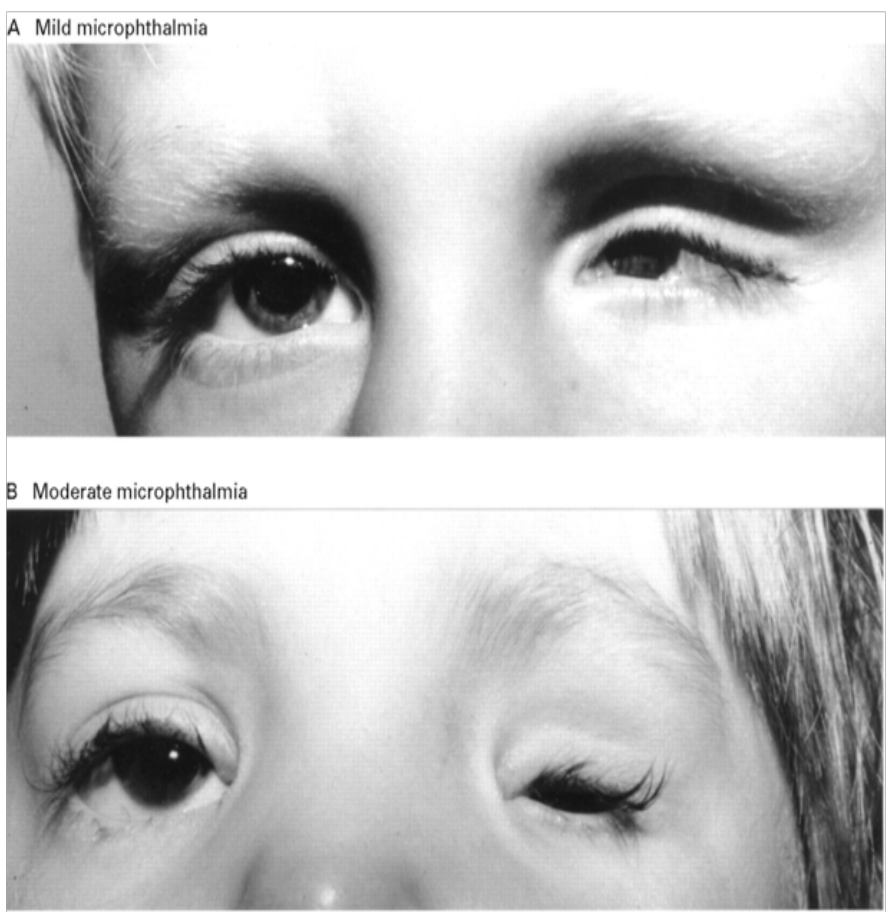

C Severe microphthalmia

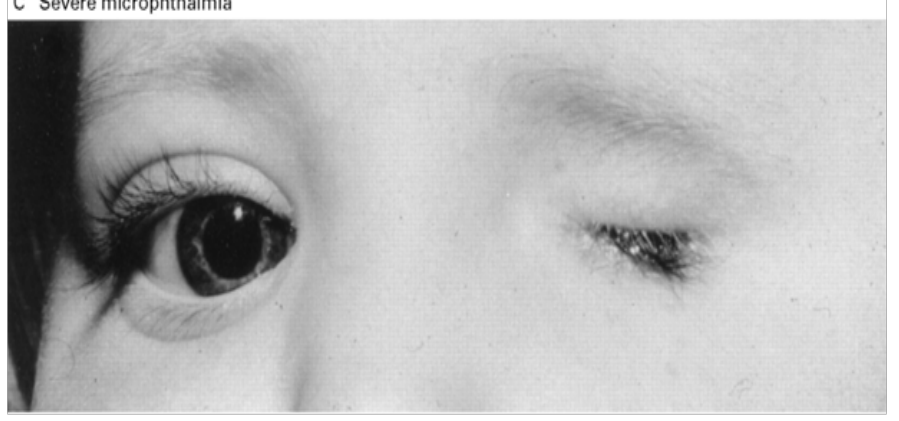

Figure 3 A) Mild microphthalmia refers to a structurally normal, small eye and has been used interchangeably with 'nanophthalmia' (though the latter is particularly used when referring to a small eye with micro-cornea, axial length). B) A phenotypic range between anophthalmia (severe microphthalmia) and microphthalmia, C) an eye with axial length $<15 \mathrm{~mm} .^{27}$

\section{Diabetes}

Diabetes occurs with an increase in blood glucose levels and, like cataract, is one of the most important causes of blindness that can be a chronic side effect of blood vessels over time. Diabetes can affect vision in various ways, including early onset of cataract, black water, and effects on the optic nerve and vascular bleeding in the eye, and eventually blindness of the individual. ${ }^{34-36}$

\section{Aids for blinds}

A device that is recognized as a helping hand and a symbol of blindness in the whole world is a white cane, but there are tools to help the blind to be active in the community. There are scanned systems that can read the age when a person moves. Most blind people make the most of their sense of hearing and feel that this is the key to many of their problems. In addition to their hearing sense, their tactile sense 
helps them to make the braille line one of the most important tools for the blind to read. The braille line works with 6points on the paper, in the form of 2 columns of three points, which combine these points together and make different languages. ${ }^{37,38}$ Another technology used by the blind is the Finger Reader, as shown in Figure 5; this device helps the blind to read information on digital devices, such that it is placed on the finger of the user and includes a number of sensors. ${ }^{39-42}$ The sequential text reading algorithm is comprised of a number of sub-algorithms concatenated in a state-machine (Figure 6), ${ }^{43}$ to accommodate for a continuous operation by a blind person. The first two states (Detect Scene and Learn Finger) are used for calibration for the higher level text extraction and tracking work states (No Line, Line Found and End of Line). Each state delivers timely audio cues to the users to inform them of the process. All states and their underlying algorithms are detailed in the following sections. The operation begins with detecting if the camera indeed is looking at a close-up view of a finger touching a contrasting paper, which is what the system expects in a typical operation. ${ }^{44}$ Once achieving a stable view, the system looks to locate the fingertip as a cursor for finding characters, words and lines. The next three states deal with finding and maintaining the working line and reading words. For finding a line, the first line or otherwise, a user may scan the page (in No Line mode) until receiving an audio cue that text has been found. While a text line is maintained, the system will stay in the Line Found state, until the user advanced to the end of the line or the line is lost (by moving too far up or down from the line or away from the paper) ${ }^{44}$ Eye-Ring consists of a fingerworn device with an embedded camera and a computation element embodied as a smartphone or computer, which is also used for speech $\mathrm{I} / \mathrm{O}$. The finger-worn device is autonomous, wireless, and includes a single button to initiate the interaction. Information from the device is transferred via Bluetooth to the computing element where it is processed. An overview of the Eye-Ring system is shown in Figure 7.45
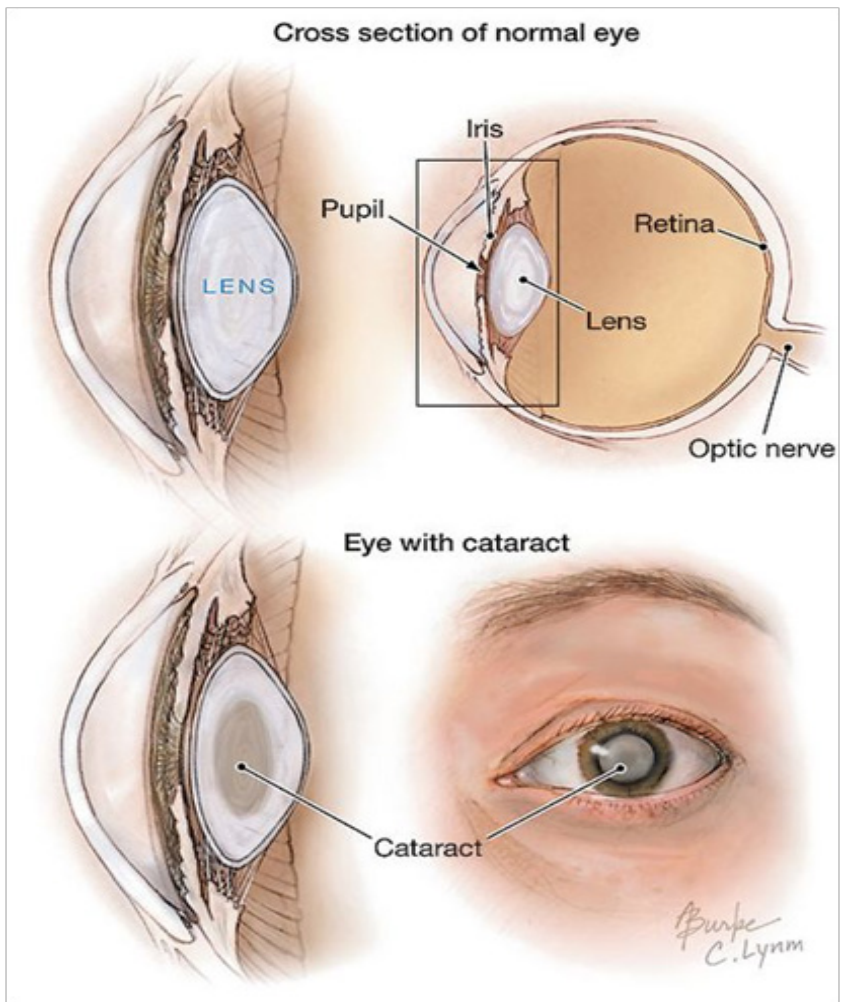

Figure 4 Cataracts are cloudy areas in the lens. They occur when certain proteins in the lens form abnormal clumps. ${ }^{33}$

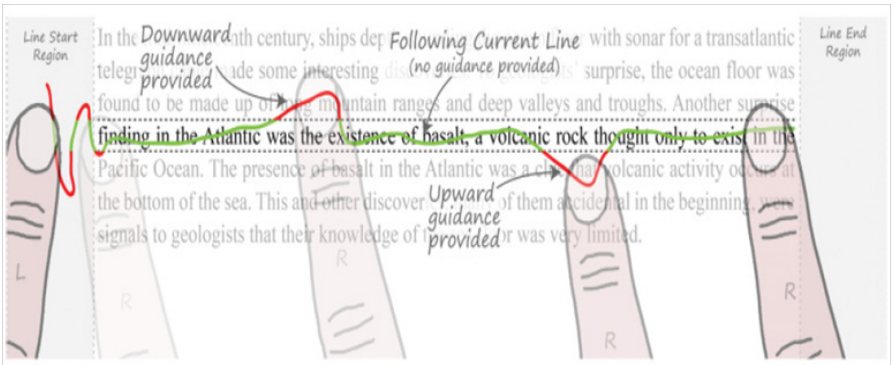

Figure 5 Reading mode interaction is bimanual. The user (I) places the right index finger in the "line start region" and moves vertically to find the start of the current line; (2) places the left index next to the right finger as an anchor; (3) traces the right finger along the line until it reaches the "line end region", (4) returns the right index finger to beside the left finger before moving down to the next line.When the right finger is directly on the line (green trace), no directional guidance is provided, but when the finger moves too high or low (red trace), audio or haptic guidance indicates which direction to move to return to the line. ${ }^{43}$

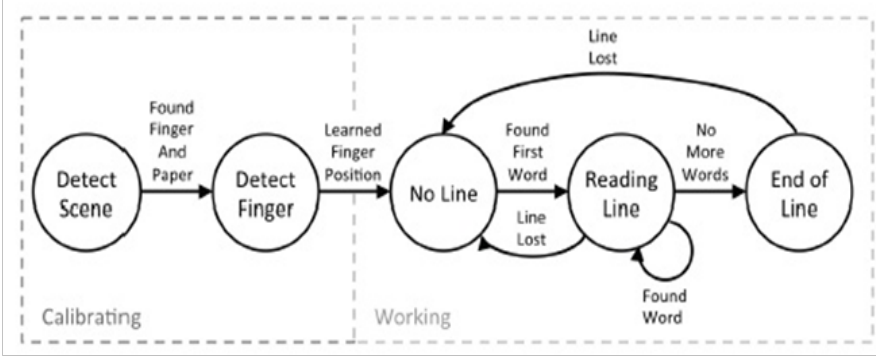

Figure 6 The process of reading the text by the finger reader device. ${ }^{43}$

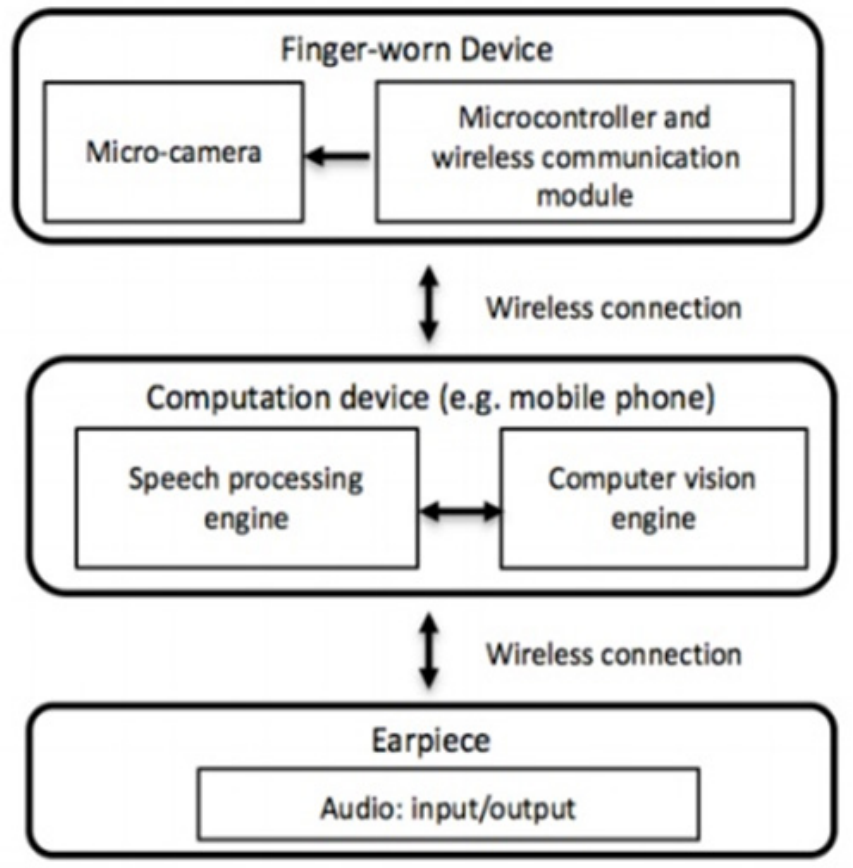

Figure 7 General Diagram Analysis of the Finger Reader. ${ }^{45}$

Another tool that helps blind people to be present on their own in the community is a money-recognition device that, as shown in Figure 8 , helps them understand the value of the money they have in their 
hands. One of the most famous brands of this device is the ibis and the function of this device is that the corner of the money is placed in the groove of the device and the user presses the button that is located above or below of the device, then a Robotic voice announces the value of money. ${ }^{46,47}$ BEP (BUREAU OF ENGRAVING AND PRINTING) provides currency readers, free of charge, to all eligible blind and visually impaired U.S. citizens and national residents. As shown in Figure 8 the device is small, compact, and runs on a triple-A battery, which is included. To use the currency reader, simply insert a U.S. bill into the reader and press the button. The device will tell the note's denomination. Guiding dogs can help many blind people. All dogs are not suitable for training as guidance dogs to help the blind, and the important reasons they refuse to nurture them as guiding dogs to help are: hypo-dysplasia, physical causes, fear, irritability, etc. ${ }^{48}$ The Labrador dog is very suitable for breeding as a guide dog. These dogs are cultivated in such a way that they operate under the command of the blind person, if they realize that they are doing something dangerous to the blind person, they will not act. ${ }^{49,50}$ Another tool that can be used to help the blind is the VibroTac, an e-assisting tool. This device can detect obstacles in different directions and prevent the blind to encounter them by using the unique vibrating devices. As shown in Figure 9 there are separate motors which are driven by the microcontroller. Each direction is obtained by the sensors and the output pulses of the sensors produce PWM pulses to drive the motors. ${ }^{51}$ Another device designed to help the blind is a wearable device that has three important functions: first, with the help of the ultrasonic sensor, provides the ability to detect objects and alerts the person in contact with the object or vice versa. Secondly, it identifies the objects by using the images taken by the camera. Third, the device declares the detected tools by the TTS engine. ${ }^{52}$ The developed mechanism, as shown in Figure 11, is tested through identical situations. Though, the collected data admitted the correct placement of the sensors and the notifications' ${ }^{53}$ Text-to-Speech, abbreviated as TTS, is a technology that converts digital text into spoken voice output. Text-to-Speech systems were first developed to aid the visually impaired. They are nowadays ubiquitous, having an extremely broad field of application ranging from voices giving directions on navigation devices to voices for public announcement systems and virtual assistants ${ }^{54}$ or intelligent sensors..$^{55-57}$

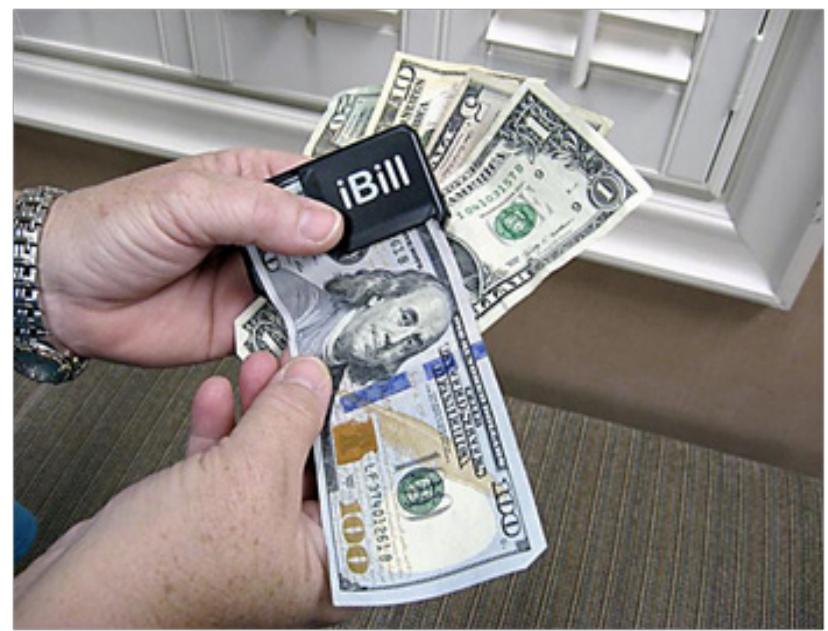

Figure 8 Second Generation iBill Talking Money Identifier. ${ }^{46}$

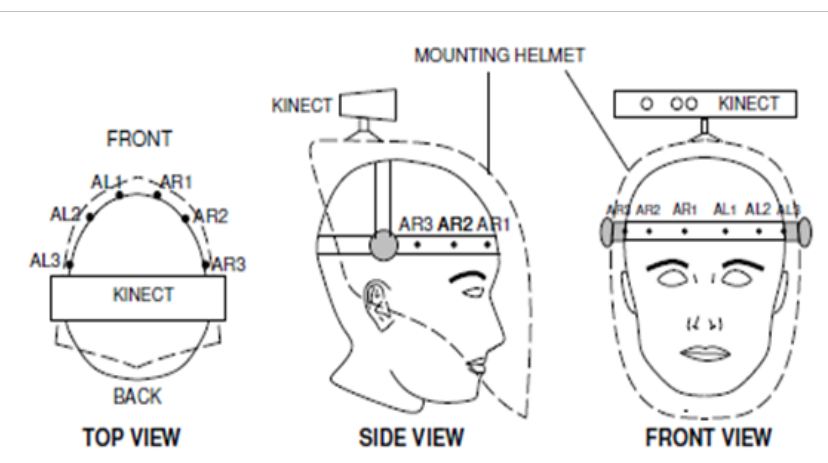

Figure 9 Wearable sensor and actuator configuration on a helmet, showing placement of the motor actuators around the forehead. ${ }^{5}$

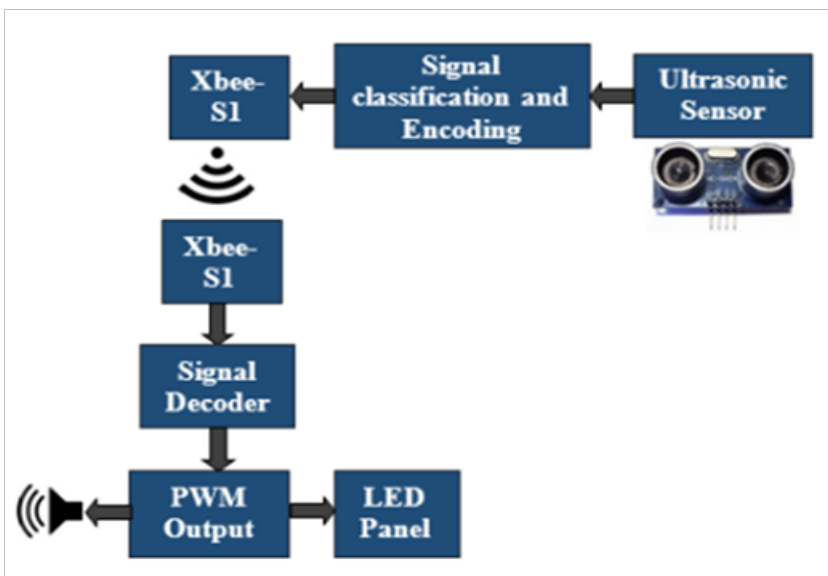

Figure 10 Ultrasonic trans-receivers (HC-SR04, Cytron Technologies, Malaysia), Arduino UNO microcontroller (Arduino, Italy), Xbee-SI transreceiver module (Digi International, USA), LEDs, pizeobuzzers and a light weight cane. ${ }^{53}$

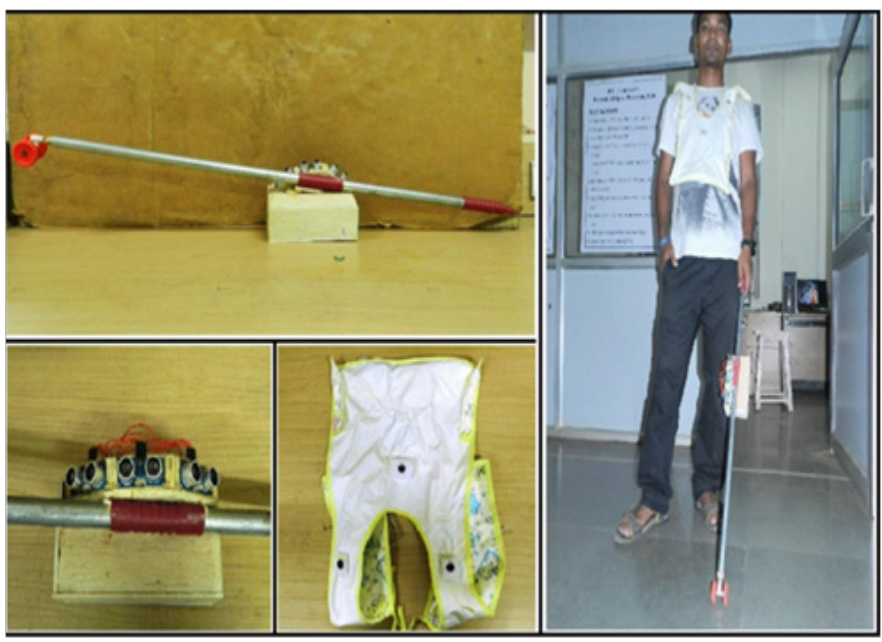

Figure I I The developed device experimental and the necessary equipment assembled on the body. ${ }^{53}$

\section{Conclusion}

The eye is one of the most sensitive parts of the body and has a very complex structure, and the damage to any part of this complex structure causes many diseases, some of which cause blindness. Blind 
people can use devices to operate their other senses like hearing and touch to do their own work in order to be able to live more easily and to be present in society, in addition to using a white cane. World is progressing in science and technology, making it easier for the blind to make various devices for life. In this study we described each part of the human eye in order to have a clear understanding of every section in the eye then we discussed the issues that any eye might encounter. But also we introduced modern methods and techniques used in the rehabilitation of the people with impaired vision. Thanks to the artificial intelligence and the robotics science, recent activities in such fields have improved the reliability and facility of the regular issues which blind people would encounter.

\section{Acknowledgments}

None.

\section{Conflicts of interest}

Author declares that there is no conflict of interest.

\section{References}

1. Simões WC, V De Lucena. Blind user wearable audio assistance for indoor navigation based on visual markers and ultrasonic obstacle detection. In 2016 IEEE International Conference on Consumer Electronics. 2016.

2. Lun Khoo W, Joey Knapp, Franklin Palmer, et al. Designing and testing wearable range-vibrotactile devices. Journal of Assistive Technologies. 20131;7(2):102-117.

3. Ng PC, Oliver JJ. Anatomy of the Eye, in Handbook of Emergency Ophthalmology. Springer; 2018:1-12.

4. https://www.allaboutvision.com/resources/anatomy.htm

5. Kolb H. Gross anatomy of the eye. 2007.

6. Galloway NR, Amoaku NR, Galloway WMK, et al. Common eye diseases and their management. Springer; 2006.

7. Binder MD, Hirokawa N, Uwe Windhorst. Windhorst, Encyclopedia of neuroscience. 2009.

8. Garces R. Glover Films Blindness in Uruguay. 2007.

9. Turan K. Foresight pays off for'Blindness' director. Los Angeles Times; 2008:15.

10. Sembulingam K, Sembulingam P. Essentials of medical physiology. JP Medical Ltd; 2012.

11. Morgan MJ, Adam A, Mollon JD. Dichromats detect colour-camouflaged objects that are not detected by trichromats. Proc $R$ Soc Lond B. 1992;248(1323):291-295.

12. Hong A, Lillian Makhathini, Mirriam Mashele, et al. Annual measles and rubella surveillance review, South Africa, 2017. 2017.

13. Jyoti MS. Shirke S, Matalia H. Congenital rubella syndrome: Global issue. Journal of Cataract \& Refractive Surgery. 2015;41(5): 1127.

14. Plotkin SA. Commentary: Is Japan deaf to mumps vaccination? The Pediatric infectious disease journal. 2009;28(3):176.

15. Adams Waldorf KM, McAdams RM. Influence of infection during pregnancy on fetal development. Reproduction. 2013: 146(5):R151-62.

16. Ackerman W, Kwiek JJ. Role of the placenta in adverse perinatal outcomes among HIV-1 seropositive women. Journal of Nippon Medical School. 2013;80(2):90-94.
17. Ahn HM, Park EA, Cho SJ, et al. The association of histological chorioamnionitis and antenatal steroids on neonatal outcome in preterm infants born at less than thirty-four weeks' gestation. Neonatology. 2012;102(4):259-264.

18. Back SA, Han BH, Luo NL, et al. Selective vulnerability of late oligodendrocyte progenitors to hypoxia-ischemia. Journal of Neuroscience. 2002;22(2):455-463.

19. Balinotti JE, Chakr VC, Tiller C, et al. Growth of lung parenchyma in infants and toddlers with chronic lung disease of infancy. American journal of respiratory and critical care medicine. 2010;181(10):1093-1097.

20. Wheatley C, Dickinson JL, Mackey DA, et al. Retinopathy of prematurity: recent advances in our understanding. British Journal of Ophthalmology. 2002;86(6):696-700.

21. Gole GA. The international classification of retinopathy of prematurity revisited. JAMA Ophthalmology. 2005;123(7):991-999.

22. Screening Examination of Premature Infants for Retinopathy of Prematurity. Pediatrics. 2013;131(1):189-195.

23. Gilbert C, Fielder A, Gordillo L, et al., Characteristics of Infants With Severe Retinopathy of Prematurity in Countries With Low, Moderate, and High Levels of Development: Implications for Screening Programs. Pediatrics. 2005;115(5):e518-e525.

24. Nihalani BR, Jani UD, Vasavada AR, et al. Cataract surgery in relative anterior microphthalmos. Ophthalmology. 2005;112(8): 1360-1367.

25. Spitznas M, Gerke E, Bateman JB. Hereditary posterior microphthalmos with papillomacular fold and high hyperopia. Archives of Ophthalmology. 1983;101(3):413-417.

26. Khairallah M, Messaoud R, Zaouali S, et al. Posterior segment changes associated with posterior microphthalmos. Ophthalmology. 2002;109(3):569-574.

27. Verma AS, Fitz Patrick DR. Anophthalmia and microphthalmia. Orphanet journal of rare diseases. 2007;2(1):47.

28. Quigley HA. Number of people with glaucoma worldwide. British journal of ophthalmology. 1996;80(5):389-393.

29. Jakobs TC. Methods and Protocols. 2018.

30. Crish SD, Dengler-Crish CM. Introduction to Glaucoma. Metabolism of Human Diseases: Organ Physiology and Pathophysiology. 2014.

31. Papaliodis GN. Cataracts, in Uveitis. Springer; 2017:337-341.

32. Annamalai A. Cataracts, in Medical Management of Psychotropic Side Effects. Springer; 2017:293-293.

33. https://www.pinterest.com/pin/487233253429504871

34. Joslin EP, Kahn CR. Joslin's Diabetes Mellitus. In: Ronald Kahn, Editor. Lippincott Williams \& Wilkins; 2005.

35. La Caille L. Eating Behavior. Medicine. 2013;38(1):S47-S55.

36. WHO. Diabetes. 1989.

37. Kipke S. Sensitive braille displays with ATC technology (active tactile control) as a tool for learning braille. International Conference on Computers for Handicapped Persons. 2008.

38. Sugirtha K, Dhanalakshmi M. Transliteration of Braille Code into Text in English Language, Computational Signal Processing and Analysis. 2018:373-381.

39. Bigham JP, Chandrika Jayant, Hanjie Ji, et al. VizWiz: nearly real-time answers to visual questions. Proceedings of the 23nd annual ACM symposium on User interface software and technology. 2010. 
40. Black AW, Lenzo KA. Flite: a small fast run-time synthesis engine. 4th ISCA Tutorial and Research Workshop (ITRW) on Speech Synthesis. 2001.

41. D Albe EF. On a type-reading optophone. Proc $R$ Soc Lond $A$. 1914;90(619):373-375

42. Hanif SM, Prevost L. Texture based Text Detection in Natural Scene Images-A Help to Blind and Visually Impaired Persons. CVHI. 2007.

43. Stearns L, Ruofei Du, Uran Oh, et al. Evaluating haptic and auditory directional guidance to assist blind people in reading printed text using finger-mounted cameras. ACM Transactions on Accessible Computing (TACCESS). 2016;9(1):1

44. Ezaki N, Bulacu M, Schomaker L. Text detection from natural scene images: towards a system for visually impaired persons. Pattern Recognition, 2004. ICPR 2004. Proceedings of the 17th International Conference on. 2004. 2004.

45. Nanayakkara S, Rai Shilkrot, Pattie Maes, et al. Eye Ring: A FingerWorn Input Device for Seamless Interactions with Our Surroundings. 2013;13-20.

46. Jayant C, Hanjie Ji, Samuel White, et al. Supporting blind photography. The proceedings of the 13th international ACM SIGACCESS conference on Computers and accessibility. 2011

47. Ott DM, Ott CB, Stoides JG. Method for verifying the denomination of currency. 1977

48. Serpell JA, Hsu Y. Development and validation of a novel method for evaluating behavior and temperament in guide dogs. Applied Animal Behaviour Science. 2001;72(4):347-364.

49. Goddard M, Beilharz R. Genetics of traits which determine the suitability of dogs as guide-dogs for the blind. Applied Animal Ethology. 1983;9(34):299-315.
50. Pfaffenberger CJ, Scott JP, Fuller JL, et al. Guide dogs for the blind: their selection, development, and training. Oxford, New York. Elsevier Scientific Publishing Company; 1976:225

51. Mann S, Jason Haung, Ryan Janzen, et al. Blind navigation with a wearable range camera and vibrotactile helmet. Proceedings of the 19th ACM international conference on Multimedia. 2011.

52. Shaik AS, Hossain G, Yeasin M. Design, development and performance evaluation of reconfigured mobile Android phone for people who are blind or visually impaired. Proceedings of the 28th ACM International Conference on Design of Communication. 2010.

53. Kumar K, Biswajeet Champaty, K. Uvanesh, et al. Development of an ultrasonic cane as a navigation aid for the blind people. 2014 International Conference on Control, Instrumentation, Communication and Computational Technologies (ICCICCT). 2014.

54. Nilesh J, Pavan Alai, Bendre MR, et al. Voice based System in Desktop and Mobile devices for blind people. International Journal of Emerging Technology and Advanced Engineering (IJETAE). 2014:404- 407.

55. Shirzadfar Hamidreza. Design and evaluation of a GMR-biosensor for magnetic characterization of biological medium. Diss. Université de Lorraine; 2014.

56. Shirzadfar Hamidreza, Mustapha Nadi, Yamada S, et al. Needle-type GMR sensor to estimate the magnetic properties of diluted ferrofluid for biomedicine application. IRBM. 2015:178-184.

57. Shirzadfar Hamidreza, Mustapha Nadi, Yamada S, et al. Characterization of a Needle-Type Probe GMR sensor for Biomedical applications. SENSORS; 2013 